\title{
Reduced two-bound core games
}

Citation for published version (APA):

Gong, D., Dietzenbacher, B., \& Peters, H. (2022). Reduced two-bound core games. Maastricht University, Graduate School of Business and Economics. GSBE Research Memoranda No. 001 https://doi.org/10.26481/umagsb.2022001

Document status and date:

Published: 20/01/2022

DOI:

10.26481/umagsb.2022001

Document Version:

Publisher's PDF, also known as Version of record

\section{Please check the document version of this publication:}

- A submitted manuscript is the version of the article upon submission and before peer-review. There can be important differences between the submitted version and the official published version of record.

People interested in the research are advised to contact the author for the final version of the publication, or visit the DOI to the publisher's website.

- The final author version and the galley proof are versions of the publication after peer review.

- The final published version features the final layout of the paper including the volume, issue and page numbers.

Link to publication

\footnotetext{
General rights rights.

- You may freely distribute the URL identifying the publication in the public portal. please follow below link for the End User Agreement:

www.umlib.nl/taverne-license

Take down policy

If you believe that this document breaches copyright please contact us at:

repository@maastrichtuniversity.nl

providing details and we will investigate your claim.
}

Copyright and moral rights for the publications made accessible in the public portal are retained by the authors and/or other copyright owners and it is a condition of accessing publications that users recognise and abide by the legal requirements associated with these

- Users may download and print one copy of any publication from the public portal for the purpose of private study or research.

- You may not further distribute the material or use it for any profit-making activity or commercial gain

If the publication is distributed under the terms of Article $25 \mathrm{fa}$ of the Dutch Copyright Act, indicated by the "Taverne" license above, 


\section{Maastricht University}

Doudou Gong, Bas Dietzenbacher, Hans Peters

Reduced two-bound core games

$\mathrm{RM} / 22 / 001$

ISSN: $2666-8807$

\section{GSBE}

Maastricht University School of Business and Economics

Graduate School of Business and Economics

P.O Box 616

NL-6200 MD Maastricht

The Netherlands 


\title{
Reduced two-bound core games
}

\author{
Doudou Gong ${ }^{1,2 *} \quad$ Bas Dietzenbacher ${ }^{2 \dagger} \quad$ Hans Peters $^{2 \ddagger}$ \\ January 19, 2022
}

\begin{abstract}
This paper studies Davis-Maschler reduced games of two-bound core games and shows that all these reduced games with respect to core elements are two-bound core games with the same pair of bounds. Based on associated reduced game properties, we axiomatically characterize the core, the nucleolus, and the egalitarian core for twobound core games. Moreover, we show that the egalitarian core for two-bound core games is single-valued and we provide an explicit expression.
\end{abstract}

Keywords: two-bound core games, reduced games, axiomatic analysis JEL classification: C71

\section{Introduction}

Cooperative games describe situations where players collaborate in coalitions and generate profits. The main issue is to allocate the profits among these collaborating players. Among the central solution concepts are the core, the nucleolus (cf. Schmeidler 1969), and the egalitarian core (cf. Arin and Iñarra 2001). The core assigns all allocations that are stable against coalitional deviations. The nucleolus assigns the allocation that lexicographically minimizes the excesses of all coalitions, which is a core allocation whenever the core is nonempty. The egalitarian core assigns all core allocations from which no other core allocation can be obtained by a transfer from a richer to a poorer player.

In this paper, we focus on two-bound core games (cf. Gong et al. 2021), where the core is nonempty and can be described by a lower bound and an upper bound on the allocations. Many games are two-bound core games, including all games with at most three players and a nonempty core, additive games, unanimity games, bankruptcy games (cf. O'Neill 1982), 1-convex games (cf. Driessen 1986), big boss games (cf. Muto et al. 1988), clan games (cf.

\footnotetext{
*E-mail: d.gong@maastrichtuniversity.nl.

†Corresponding author. E-mail: b.dietzenbacher@maastrichtuniversity.nl.

${ }_{\ddagger}^{\ddagger}$ E-mail: h.peters@maastrichtuniversity.nl.

${ }^{1}$ School of Mathematics and Statistics, Northwestern Polytechnical University, Xi'an, 710072, China.

${ }^{2}$ Department of Quantitative Economics, Maastricht University, Maastricht, 6200 MD, The Netherlands.
} 
Potters et al. 1989), compromise stable games (cf. Quant et al. 2005), and reasonable stable games (cf. Dietzenbacher 2018).

In particular, we study Davis-Maschler reduced games of two-bound core games and show that all these reduced games with respect to core elements are two-bound core games. Moreover, the core of these reduced games can be described by the same pair of bounds. A solution satisfies the bilateral reduced game property (cf. Davis and Maschler 1965) if the restriction of each allocation assigned to the original game is consistently assigned to all reduced games with two players. A solution satisfies the converse reduced game property (cf. Davis and Maschler 1965) if all allocations for which each two-player restriction is assigned to the corresponding reduced game are assigned to the original game. Using the bilateral reduced game property and the converse reduced game property, we axiomatically characterize the core, the nucleolus, and the egalitarian core for two-bound core games. Moreover, we show that the egalitarian core for two-bound core games is single-valued and we provide an explicit expression.

The remainder of this paper is organized as follows. Section 2 introduces preliminary definitions and notations about cooperative games. Section 3 studies Davis-Maschler reduced games of two-bound core games and axiomatically characterize the core. Sections 4 and 5 characterize the nucleolus and the egalitarian core, respectively. Section 6 concludes.

\section{Preliminaries}

Let $N$ be a nonempty and finite set of players and let $2^{N}$ be the collection of all subsets of $N$. For $x, y \in \mathbb{R}^{N}, x \geq y$ denotes $x_{i} \geq y_{i}$ for all $i \in N$, and $x>y$ denotes $x_{i}>y_{i}$ for all $i \in N$. The notations $\leq$ and $<$ are defined analogously. We denote $x+y=\left(x_{i}+y_{i}\right)_{i \in N}$, $x-y=\left(x_{i}-y_{i}\right)_{i \in N},[x, y]=\left\{z \in \mathbb{R}^{N} \mid x \leq z \leq y\right\}, \lambda x=\left(\lambda x_{i}\right)_{i \in N}$ for all $\lambda \in \mathbb{R}$, and $x_{S}=\left(x_{i}\right)_{i \in S}$ for all $S \in 2^{N} \backslash\{\emptyset\}$.

A cooperative game with transferable utility (a game, for short) is a pair $(N, v)$, where $v: 2^{N} \rightarrow \mathbb{R}$ is the characteristic function assigning to each coalition $S \in 2^{N}$ its worth, with $v(\emptyset)=0$. The set of all games with player set $N$ is denoted by $\Gamma^{N}$. For simplicity, we write $v \in \Gamma^{N}$ rather than $(N, v) \in \Gamma^{N}$.

Let $v \in \Gamma^{N}$. The pre-imputation set of $v$ is the set

$$
X(v)=\left\{x \in \mathbb{R}^{N} \mid \sum_{i \in N} x_{i}=v(N)\right\}
$$

and the core of $v$ is the set

$$
C(v)=\left\{x \in X(v) \mid \forall_{S \in 2^{N}}: \sum_{i \in S} x_{i} \geq v(S)\right\} .
$$


The set of all games with nonempty core and player set $N$ is denoted by $\Gamma_{b}^{N}$. A game $v \in \Gamma^{N}$ is convex (cf. Shapley 1971) if $v(S)+v(T) \leq v(S \cup T)+v(S \cap T)$ for all $S, T \in 2^{N}$. The set of all convex games with player set $N$ is denoted by $\Gamma_{c}^{N}$. It is known that $\Gamma_{c}^{N} \subseteq \Gamma_{b}^{N}$.

A game $v \in \Gamma_{b}^{N}$ is a two-bound core game (cf. Gong et al. 2021) if there exist $l, u \in \mathbb{R}^{N}$ such that $C(v)=[l, u] \cap X(v)$, which is equivalent to $C(v)=\left[l^{*}(v), u^{*}(v)\right] \cap X(v)$, where $l_{i}^{*}(v)=\min _{x \in C(v)} x_{i}$ and $u_{i}^{*}(v)=\max _{x \in C(v)} x_{i}$ for all $i \in N$. The bounds $l^{*}$ and $u^{*}$ were also studied by Bondareva and Driessen (1994). The set of all two-bound core games with player set $N$ is denoted by $\Gamma_{t}^{N}$.

A solution $\varphi$ on a domain of games assigns to each game $v$ in this domain a nonempty set $\varphi(v) \subseteq X(v)$. Note that $\varphi(v)=\{v(N)\}$ for each game $v$ with one player. A solution $\varphi$ on a domain of games is single-valued if $|\varphi(v)|=1$ for each $v$ in this domain. For a single-valued solution $\varphi$ on a domain of games and a game $v$ in this domain, $\varphi(v)$ is often identified with its unique element.

\section{Reduced games and the core}

In this section, we study Davis-Maschler reduced games of two-bound core games, and axiomatically characterize the core for two-bound core games. First, we show that the core of a two-bound core game is equal to the core of a convex game.

\section{Theorem 1}

Let $v \in \Gamma_{t}^{N}$. Then there exists $\widehat{v} \in \Gamma_{c}^{N}$ such that $C(\widehat{v})=C(v)$.

Proof. Let $l, u \in \mathbb{R}^{N}$ be such that $C(v)=[l, u] \cap X(v)$. Define $\widehat{v} \in \Gamma^{N}$ by

$$
\widehat{v}(S)=\max \left\{\sum_{i \in S} l_{i}, v(N)-\sum_{i \in N \backslash S} u_{i}\right\} \text { for all } S \in 2^{N} .
$$

Gong et al. (2021) showed that $C(\widehat{v})=C(v)$, which implies that $\widehat{v} \in \Gamma_{t}^{N}$. For all $S, T \in 2^{N}$,

$$
\begin{aligned}
& \widehat{v}(S)+\widehat{v}(T) \\
= & \max \left\{\sum_{i \in S} l_{i}, v(N)-\sum_{i \in N \backslash S} u_{i}\right\}+\max \left\{\sum_{i \in T} l_{i}, v(N)-\sum_{i \in N \backslash T} u_{i}\right\} \\
= & \max \left\{\sum_{i \in S} l_{i}+\sum_{i \in T} l_{i}, v(N)+\sum_{i \in S} l_{i}-\sum_{i \in N \backslash T} u_{i},\right. \\
& \left.v(N)+\sum_{i \in T} l_{i}-\sum_{i \in N \backslash S} u_{i}, 2 v(N)-\sum_{i \in N \backslash S} u_{i}-\sum_{i \in N \backslash T} u_{i}\right\}
\end{aligned}
$$




$$
\begin{aligned}
& \leq \max \left\{\sum_{i \in S \cup T} l_{i}+\sum_{i \in S \cap T} l_{i}, v(N)+\sum_{i \in S} l_{i}-\sum_{i \in N \backslash T} u_{i}+\sum_{i \in S \backslash T}\left(u_{i}-l_{i}\right),\right. \\
& \left.v(N)+\sum_{i \in T} l_{i}-\sum_{i \in N \backslash S} u_{i}+\sum_{i \in T \backslash S}\left(u_{i}-l_{i}\right), 2 v(N)-\sum_{i \in N \backslash S} u_{i}-\sum_{i \in N \backslash T} u_{i}\right\} \\
& =\max \left\{\sum_{i \in S \cup T} l_{i}+\sum_{i \in S \cap T} l_{i}, v(N)+\sum_{i \in S \cap T} l_{i}-\sum_{i \in N \backslash(S \cup T)} u_{i},\right. \\
& \left.v(N)+\sum_{i \in S \cap T} l_{i}-\sum_{i \in N \backslash(S \cup T)} u_{i}, 2 v(N)-\sum_{i \in N \backslash(S \cup T)} u_{i}-\sum_{i \in N \backslash(S \cap T)} u_{i}\right\} \\
& \leq \max \left\{\sum_{i \in S \cup T} l_{i}, v(N)-\sum_{i \in N \backslash(S \cup T)} u_{i}\right\}+\max \left\{\sum_{i \in S \cap T} l_{i}, v(N)-\sum_{i \in N \backslash(S \cap T)} u_{i}\right\} \\
& =\widehat{v}(S \cup T)+\widehat{v}(S \cap T) .
\end{aligned}
$$

Hence, $\widehat{v} \in \Gamma_{c}^{N}$.

The reduced game (cf. Davis and Maschler 1965) of $v \in \Gamma_{t}^{N}$ on $T \in 2^{N} \backslash\{\emptyset\}$ with respect to $x \in \mathbb{R}^{N}$, denoted by $v_{T}^{x} \in \Gamma^{T}$, is defined by

$$
v_{T}^{x}(S)= \begin{cases}v(N)-\sum_{i \in N \backslash T} x_{i} & \text { if } S=T, \\ \max _{Q \subseteq N \backslash T}\left\{v(S \cup Q)-\sum_{i \in Q} x_{i}\right\} & \text { if } S \in 2^{T} \backslash\{\emptyset, T\}, \\ 0 & \text { if } S=\emptyset .\end{cases}
$$

It turns out that all reduced games of two-bound core games with respect to core elements are two-bound core games. Moreover, the core of these reduced games can be described by the same pair of bounds.

\section{Theorem 2}

Let $v \in \Gamma_{t}^{N}, T \in 2^{N} \backslash\{\emptyset\}, x \in C(v)$, and let $l, u \in \mathbb{R}^{N}$ be such that $C(v)=[l, u] \cap X(v)$. Then

$$
C\left(v_{T}^{x}\right)=\left[l_{T}, u_{T}\right] \cap X\left(v_{T}^{x}\right) .
$$

Proof. Let $y \in C\left(v_{T}^{x}\right)$. Then

$$
\sum_{i \in T} y_{i}+\sum_{i \in N \backslash T} x_{i}=v_{T}^{x}(T)+\sum_{i \in N \backslash T} x_{i}=v(N)-\sum_{i \in N \backslash T} x_{i}+\sum_{i \in N \backslash T} x_{i}=v(N) .
$$


For all $S \in 2^{N} \backslash\{\emptyset, N\}$,

$$
\begin{aligned}
\sum_{i \in S \cap T} y_{i}+\sum_{i \in S \backslash T} x_{i} & \geq v_{T}^{x}(S \cap T)+\sum_{i \in S \backslash T} x_{i} \\
& =\max _{Q \subseteq N \backslash T}\left\{v((S \cap T) \cup Q)-\sum_{i \in Q} x_{i}\right\}+\sum_{i \in S \backslash T} x_{i} \\
& \geq v(S)-\sum_{i \in S \backslash T} x_{i}+\sum_{i \in S \backslash T} x_{i} \\
& =v(S) .
\end{aligned}
$$

This means that $\left(y, x_{N \backslash T}\right) \in C(v)$, so $\left(y, x_{N \backslash T}\right) \in[l, u] \cap X(v)$, which implies that $y \in$ $\left[l_{T}, u_{T}\right] \cap X\left(v_{T}^{x}\right)$. Hence, $C\left(v_{T}^{x}\right) \subseteq\left[l_{T}, u_{T}\right] \cap X\left(v_{T}^{x}\right)$.

Let $y \in\left[l_{T}, u_{T}\right] \cap X\left(v_{T}^{x}\right)$. Then $\left(y, x_{N \backslash T}\right) \in[l, u] \cap X(v)$, so $\left(y, x_{N \backslash T}\right) \in C(v)$. Let $S \in 2^{T} \backslash\{\emptyset, T\}$. For all $Q \subseteq N \backslash T$,

$$
\sum_{i \in S} y_{i}=\sum_{i \in S} y_{i}+\sum_{i \in Q} x_{i}-\sum_{i \in Q} x_{i} \geq v(S \cup Q)-\sum_{i \in Q} x_{i}
$$

so

$$
\sum_{i \in S} y_{i} \geq \max _{Q \subseteq N \backslash T}\left\{v(S \cup Q)-\sum_{i \in Q} x_{i}\right\}=v_{T}^{x}(S) .
$$

This implies that $y \in C\left(v_{T}^{x}\right)$. Hence, $\left[l_{T}, u_{T}\right] \cap X\left(v_{T}^{x}\right) \subseteq C\left(v_{T}^{x}\right)$.

A solution satisfies the bilateral reduced game property if the restriction of each preimputation assigned to the original game is consistently assigned to all reduced games with two players. The converse reduced game property requires that if each two-player restriction of a pre-imputation is assigned to the corresponding reduced game, then this pre-imputation is assigned to the original game.

Bilateral reduced game property (cf. Davis and Maschler 1965)

For all $v \in \Gamma_{t}^{N}$, all $T \in 2^{N}$ with $|T|=2$, and all $x \in \varphi(v)$, we have $v_{T}^{x} \in \Gamma_{t}^{T}$ and $x_{T} \in \varphi\left(v_{T}^{x}\right)$.

Converse reduced game property (cf. Davis and Maschler 1965)

For all $v \in \Gamma_{t}^{N}$ and all $x \in X(v)$, if $v_{T}^{x} \in \Gamma_{t}^{T}$ and $x_{T} \in \varphi\left(v_{T}^{x}\right)$ for all $T \in 2^{N}$ with $|T|=2$, then $x \in \varphi(v)$.

By requiring the solution to assign the core to all two-bound core games with two players, we obtain an axiomatic characterization of the core for two-bound core games using the bilateral reduced game property and the converse reduced game property.

Unanimity (cf. Peleg 1986)

For all $v \in \Gamma_{t}^{N}$ with $|N|=2$, we have $\varphi(v)=\left\{x \in X(v) \mid \forall_{i \in N}: x_{i} \geq v(\{i\})\right\}$. 


\section{Theorem 3}

The core is the unique solution for two-bound core games satisfying unanimity, the bilateral reduced game property, and the converse reduced game property.

Proof. Clearly, the core satisfies unanimity. To prove that the core satisfies the bilateral reduced game property, let $v \in \Gamma_{t}^{N}$, let $T \in 2^{N}$ with $|T|=2$, let $x \in C(v)$, and let $l, u \in \mathbb{R}^{N}$ be such that $C(v)=[l, u] \cap X(v)$. By Theorem 2, $C\left(v_{T}^{x}\right)=\left[l_{T}, u_{T}\right] \cap X\left(v_{T}^{x}\right)$. In view of $x_{T} \in\left[l_{T}, u_{T}\right] \cap X\left(v_{T}^{x}\right)$, this implies that $v_{T}^{x} \in \Gamma_{t}^{T}$ and $x_{T} \in C\left(v_{T}^{x}\right)$. Hence, the core satisfies the bilateral reduced game property.

To prove that the core satisfies the converse reduced game property, let $v \in \Gamma_{t}^{N}$ and let $x \in X(v)$ be such that $v_{T}^{x} \in \Gamma_{t}^{T}$ and $x_{T} \in C\left(v_{T}^{x}\right)$ for all $T \in 2^{N}$ with $|T|=2$. Let $S \in 2^{N} \backslash\{\emptyset, N\}$ and let $j \in N \backslash S$. For all $i \in S$,

$$
x_{i} \geq v_{\{i, j\}}^{x}(\{i\})=\max _{Q \subseteq N \backslash\{i, j\}}\left\{v(\{i\} \cup Q)-\sum_{k \in Q} x_{i}\right\} \geq v(S)-\sum_{k \in S \backslash\{i\}} x_{k},
$$

so $\sum_{i \in S} x_{i} \geq v(S)$. This implies that $x \in C(v)$. Hence, the core satisfies the converse reduced game property.

To prove uniqueness, let $\varphi$ be a solution for two-bound core games satisfying unanimity, the bilateral reduced game property, and the converse reduced game property. We show that $\varphi(v)=C(v)$ for all $v \in \Gamma_{t}^{N}$. By unanimity, $\varphi(v)=C(v)$ for all $v \in \Gamma_{t}^{N}$ with $|N| \leq 2$. Let $v \in \Gamma_{t}^{N}$ with $|N| \geq 3$.

Let $x \in \varphi(v)$. By the bilateral reduced game property of $\varphi, v_{T}^{x} \in \Gamma_{t}^{T}$ and $x_{T} \in \varphi\left(v_{T}^{x}\right)$ for all $T \in 2^{N}$ with $|T|=2$, so $x_{T} \in C\left(v_{T}^{x}\right)$ for all $T \in 2^{N}$ with $|T|=2$. By the converse reduced game property of the core, this implies that $x \in C(v)$. Hence, $\varphi(v) \subseteq C(v)$.

Let $x \in C(v)$. By the bilateral reduced game property of the core, $v_{T}^{x} \in \Gamma_{t}^{T}$ and $x_{T} \in$ $C\left(v_{T}^{x}\right)$ for all $T \in 2^{N}$ with $|T|=2$, so $x_{T} \in \varphi\left(v_{T}^{x}\right)$ for all $T \in 2^{N}$ with $|T|=2$. By the converse reduced game property of $\varphi$, this implies that $x \in \varphi(v)$. Hence, $C(v) \subseteq \varphi(v)$.

\section{The nucleolus}

In this section, we axiomatically characterize the nucleolus for two-bound core games using the Davis-Maschler reduced game properties. The nucleolus $\eta$ (cf. Schmeidler 1969) is the single-valued solution that assigns to each game with nonempty core the unique core element that lexicographically minimizes the maximal excesses of all coalitions. Gong et al. (2021) provided an explicit expression of the nucleolus of a two-bound core game $v \in \Gamma_{t}^{N}$, with 
$C(v)=[l, u] \cap X(v)$ for $l, u \in \mathbb{R}^{N}$, which is equivalent to

$$
\eta_{i}(v)= \begin{cases}l_{i}+\min \left\{\frac{1}{2}\left(u_{i}-l_{i}\right), \lambda\right\} & \text { if } \frac{1}{2} \sum_{i \in N}\left(u_{i}+l_{i}\right) \geq v(N), \\ l_{i}+\max \left\{\frac{1}{2}\left(u_{i}-l_{i}\right), u_{i}-l_{i}-\lambda\right\} & \text { if } \frac{1}{2} \sum_{i \in N}\left(u_{i}+l_{i}\right) \leq v(N),\end{cases}
$$

for all $i \in N$, where $\lambda \in \mathbb{R}$ is such that $\sum_{i \in N} \eta_{i}(v)=v(N)$.

By requiring the solution to assign the nucleolus to all two-bound core games with two players, we obtain an axiomatic characterization of the nucleolus for two-bound core games using the bilateral reduced game property.

Standardness (cf. Aumann and Maschler 1985)

For all $v \in \Gamma_{t}^{N}$ with $|N|=2$ and all $i \in N$, we have

$$
\varphi_{i}(v)=v(\{i\})+\frac{1}{2}(v(N)-v(\{i\})-v(N \backslash\{i\})) .
$$

\section{Theorem 4}

The nucleolus is the unique solution for two-bound core games satisfying standardness and the bilateral reduced game property.

Proof. It is known that the nucleolus satisfies standardness. To prove that the nucleolus satisfies the bilateral reduced game property and the converse reduced game property (used in the uniqueness part), let $v \in \Gamma_{t}^{N}$, let $l, u \in \mathbb{R}^{N}$ be such that $C(v)=[l, u] \cap X(v)$, and let $x \in X(v)$. By Theorem 1 1 there exists $\widehat{v} \in \Gamma_{c}^{N}$ such that $C(\widehat{v})=C(v)$. Expression (1) implies that $\eta(\widehat{v})=\eta(v)$. Maschler et al. (1971) showed that the convexity of $\widehat{v}$ implies that

$$
\eta(\widehat{v})=\left\{x \in X(\widehat{v}) \mid \forall_{i, j \in N, i \neq j}: s_{i j}^{x}(\widehat{v})=s_{j i}^{x}(\widehat{v})\right\},
$$

where $s_{i j}^{x}(\widehat{v})=\max _{S \in 2^{N}: i \in S, j \notin S}\left\{\widehat{v}(S)-\sum_{k \in S} x_{k}\right\}$ for all $i, j \in N$ with $i \neq j$. For all $i, j \in N$ with $i \neq j$,

$$
\begin{aligned}
& s_{i j}^{x_{\{i, j\}}}\left(\widehat{v}_{\{i, j\}}^{x}\right)=\widehat{v}_{\{i, j\}}^{x}(\{i\})-x_{i} \\
& =\max _{Q \subseteq N \backslash\{i, j\}}\left\{\widehat{v}(Q \cup\{i\})-\sum_{k \in Q} x_{k}\right\}-x_{i} \\
& =\max _{S \in 2^{N}: i \in S, j \notin S}\left\{\widehat{v}(S)-\sum_{k \in S} x_{k}\right\} \\
& =s_{i j}^{x}(\widehat{v}) \text {. }
\end{aligned}
$$


This implies that $x=\eta(\widehat{v})$ if and only if $\widehat{v}_{T}^{x} \in \Gamma_{t}^{T}$ and $x_{T}=\eta\left(\widehat{v}_{T}^{x}\right)$ for all $T \in 2^{N}$ with $|T|=2$. By Theorem 2 if $x \in C(v)$ and $C(v)=C(\widehat{v})$, then $C\left(\widehat{v}_{T}^{x}\right)=C\left(v_{T}^{x}\right)=\left[l_{T}, u_{T}\right] \cap X\left(v_{T}^{x}\right)$ for all $T \in 2^{N}$ with $|T|=2$. By Theorem 3, $x \in C(v)$ if and only if $v_{T}^{x} \in \Gamma_{t}^{T}$ and $x_{T} \in C\left(v_{T}^{x}\right)$ for all $T \in 2^{N}$ with $|T|=2$. Together, this implies that $x=\eta(v)$ if and only if $v_{T}^{x} \in \Gamma_{t}^{T}$ and $x_{T}=\eta\left(v_{T}^{x}\right)$ for all $T \in 2^{N}$ with $|T|=2$. Hence, the nucleolus satisfies the bilateral reduced game property and the converse reduced game property.

To prove uniqueness, let $\varphi$ be a solution for two-bound core games satisfying standardness and the bilateral reduced game property. We show that $\varphi(v)=\eta(v)$ for all $v \in \Gamma_{t}^{N}$. By standardness, $\varphi(v)=\eta(v)$ for all $v \in \Gamma_{t}^{N}$ with $|N| \leq 2$. Let $v \in \Gamma_{t}^{N}$ with $|N| \geq 3$ and let $x \in \varphi(v)$. By the bilateral reduced game property of $\varphi, v_{T}^{x} \in \Gamma_{t}^{T}$ and $x_{T} \in \varphi\left(v_{T}^{x}\right)$ for all $T \in 2^{N}$ with $|T|=2$, so $x_{T}=\eta\left(v_{T}^{x}\right)$ for all $T \in 2^{N}$ with $|T|=2$. By the converse reduced game property of the nucleolus, this implies that $x=\eta(v)$. Hence, $\varphi(v)=\eta(v)$.

\section{The egalitarian core}

In this section, we axiomatically characterize the egalitarian core for two-bound core games using the Davis-Maschler reduced game properties. The egalitarian core EC (cf. Arin and Iñarra 2001) of a game $v \in \Gamma^{N}$ is defined by

$$
E C(v)=\left\{x \in C(v) \mid \forall_{i, j \in N: x_{i}>x_{j}}: s_{i j}^{x}(v)=0\right\},
$$

where, as in the proof of Theorem 4

$$
s_{i j}^{x}(v)=\max _{S \in 2^{N}: i \in S, j \notin S}\left\{v(S)-\sum_{k \in S} x_{k}\right\} .
$$

The egalitarian core consists of all core elements from which no other core element can be obtained by a transfer from a richer to a poorer player. It can be shown that if two games have equal cores, then the games have equal egalitarian cores. Arin and Iñarra (2001) showed that the egalitarian core is single-valued for convex games. Remarkably, we show that the egalitarian core is also single-valued for two-bound core games and we provide an explicit expression.

\section{Theorem 5}

The egalitarian core for two-bound core games is single-valued and, for all $v \in \Gamma_{t}^{N}$, given by

$$
E C_{i}(v)= \begin{cases}l_{i}^{*}(v) & \text { if } \lambda \leq l_{i}^{*}(v), \\ \lambda & \text { if } l_{i}^{*}(v) \leq \lambda \leq u_{i}^{*}(v), \\ u_{i}^{*}(v) & \text { if } \lambda \geq u_{i}^{*}(v),\end{cases}
$$

for all $i \in N$, where $\lambda \in \mathbb{R}$ is such that $\sum_{i \in N} E C_{i}(v)=v(N)$. 
Proof. Let $v \in \Gamma_{t}^{N}$. By Theorem 1, there exists $\widehat{v} \in \Gamma_{c}^{N}$ such that $C(\widehat{v})=C(v)$. This implies that $E C(\widehat{v})=E C(v)$. Since the egalitarian core is single-valued for convex games, $E C(v)$ is single-valued. Define $x \in \mathbb{R}^{N}$ by $x_{i}=\min \left\{\max \left\{l_{i}^{*}(v), \lambda\right\}, u_{i}^{*}(v)\right\}$ for all $i \in N$, where $\lambda \in \mathbb{R}$ is such that $\sum_{i \in N} x_{i}=v(N)$. Then $x \in\left[l^{*}(v), u^{*}(v)\right] \cap X(v)$, so $x \in C(v)$. Let $i, j \in N$ be such that $x_{i}>x_{j}$. Then $x_{i}=l_{i}^{*}(v)$ or $x_{j}=u_{i}^{*}(v)$. Suppose for the sake of contradiction that $s_{i j}^{x}(v) \neq 0$. Then $s_{i j}^{x}(v)<0$, so $v(S)<\sum_{k \in S} x_{k}$ for all $S \in 2^{N}$ with $i \in S$ and $j \notin S$. Let $0<\varepsilon<-s_{i j}^{x}(v)$. Define $x^{\prime} \in \mathbb{R}^{N}$ by $x_{i}^{\prime}=x_{i}-\varepsilon, x_{j}^{\prime}=x_{j}+\varepsilon$, and $x_{k}^{\prime}=x_{k}$ for all $k \in N \backslash\{i, j\}$. Then $x^{\prime} \in C(v)$, which contradicts the definition of $l_{i}^{*}(v)$ or $u_{j}^{*}(v)$.

By requiring the solution to assign the egalitarian core to all games with two players, Arin and Iñarra (2001) obtained an axiomatic characterization of the egalitarian core for convex games in conjunction with the bilateral reduced game property and the converse reduced game property. We obtain a similar axiomatic characterization of the egalitarian core for two-bound core games without requiring the converse reduced game property.

Constrained egalitarianism (cf. Dutta 1990)

For all $v \in \Gamma_{t}^{N}$ with $|N|=2$ and all $i \in N$, we have

$$
\varphi_{i}(v)= \begin{cases}\max \left\{v(\{i\}), \frac{1}{2} v(N)\right\} & \text { if } v(\{i\}) \geq v(N \backslash\{i\}), \\ v(N)-\varphi_{N \backslash\{i\}}(v) & \text { if } v(\{i\}) \leq v(N \backslash\{i\}) .\end{cases}
$$

\section{Theorem 6}

The egalitarian core is the unique solution for two-bound core games satisfying constrained egalitarianism and the bilateral reduced game property.

Proof. It is known that the egalitarian core satisfies constrained egalitarianism. To prove that the egalitarian core satisfies the bilateral reduced game property and the converse reduced game property (used in the uniqueness part), let $v \in \Gamma_{t}^{N}$ and let $x \in X(v)$. By Theorem 3 , $x \in C(v)$ if and only if $v_{T}^{x} \in \Gamma_{t}^{T}$ and $x_{T} \in C\left(v_{T}^{x}\right)$ for all $T \in 2^{N}$ with $|T|=2$. For all $i, j \in N$ with $i \neq j$,

$$
\begin{aligned}
& s_{i j}^{x_{\{i, j\}}}\left(v_{\{i, j\}}^{x}\right)=v_{\{i, j\}}^{x}(\{i\})-x_{i} \\
& =\max _{Q \subseteq N \backslash\{i, j\}}\left\{v(Q \cup\{i\})-\sum_{k \in Q} x_{k}\right\}-x_{i} \\
& =\max _{S \in 2^{N}: i \in S, j \notin S}\left\{v(S)-\sum_{k \in S} x_{k}\right\} \\
& =s_{i j}^{x}(v) \text {. }
\end{aligned}
$$


This implies that $x=E C(v)$ if and only if $v_{T}^{x} \in \Gamma_{t}^{T}$ and $x_{T}=E C\left(v_{T}^{x}\right)$ for all $T \in 2^{N}$ with $|T|=2$. Hence, the egalitarian core satisfies the bilateral reduced game property and the converse reduced game property.

To prove uniqueness, let $\varphi$ be a solution for two-bound core games satisfying constrained egalitarianism and the bilateral reduced game property. We show that $\varphi(v)=E C(v)$ for all $v \in \Gamma_{t}^{N}$. By constrained egalitarianism, $\varphi(v)=E C(v)$ for all $v \in \Gamma_{t}^{N}$ with $|N| \leq 2$. Let $v \in \Gamma_{t}^{N}$ with $|N| \geq 3$ and let $x \in \varphi(v)$. By the bilateral reduced game property of $\varphi$, $v_{T}^{x} \in \Gamma_{t}^{T}$ and $x_{T} \in \varphi\left(v_{T}^{x}\right)$ for all $T \in 2^{N}$ with $|T|=2$, so $x_{T}=E C\left(v_{T}^{x}\right)$ for all $T \in 2^{N}$ with $|T|=2$. By the converse reduced game property of the egalitarian core, this implies that $x=E C(v)$. Hence, $\varphi(v)=E C(v)$.

\section{Concluding remarks}

In this paper, we axiomatically characterized the core, the nucleolus, and the egalitarian core for two-bound core games using the Davis-Maschler reduced game properties. In fact, it can be shown that these solutions satisfy the stronger reduced game property which requires that the restriction of each pre-imputation assigned to the original game is consistently assigned to all reduced games (not only with two players), but the weaker bilateral reduced game property suffices in the axiomatic characterizations. To show that the properties in these axiomatic characterizations are independent, we introduce the following additional solutions.

A solution that satisfies unanimity and the converse reduced game property, but does not satisfy the bilateral reduced game property, is the solution $\widehat{X}$, which is for all $v \in \Gamma_{t}^{N}$ defined by

$$
\widehat{X}(v)= \begin{cases}C(v) & \text { if }|N| \leq 2 \\ X(v) & \text { if }|N| \geq 3\end{cases}
$$

A solution that satisfies unanimity and the bilateral reduced game property, but does not satisfy the converse reduced game property, is the solution $\widehat{C}$, which is for all $v \in \Gamma_{t}^{N}$ defined by

$$
\widehat{C}(v)= \begin{cases}C(v) & \text { if }|N| \leq 2, \\ \eta(v) & \text { if }|N| \geq 3 .\end{cases}
$$

A solution that satisfies standardness, but does not satisfy the bilateral reduced game property, is the solution $\widehat{\eta}$, which is for all $v \in \Gamma_{t}^{N}$ defined by

$$
\widehat{\eta}(v)= \begin{cases}\eta(v) & \text { if }|N| \leq 2, \\ X(v) & \text { if }|N| \geq 3\end{cases}
$$


A solution that satisfies constrained egalitarianism, but does not satisfy the bilateral reduced game property, is the solution $\widehat{E C}$, which is for all $v \in \Gamma_{t}^{N}$ defined by

$$
\widehat{E C}(v)= \begin{cases}E C(v) & \text { if }|N| \leq 2 \\ X(v) & \text { if }|N| \geq 3\end{cases}
$$

An overview of these solutions, their properties, and the axiomatic characterizations is presented in the following table.

\begin{tabular}{l||c|c|c||c|c|c|c} 
& $C$ & $\eta$ & $E C$ & $\widehat{X}$ & $\widehat{C}$ & $\widehat{\eta}$ & $\widehat{E C}$ \\
\hline unanimity & $+^{*}$ & - & - & + & + & - & - \\
standardness & - & $+^{*}$ & - & - & - & + & - \\
constrained egalitarianism & - & - & $+^{*}$ & - & - & - & + \\
\hline bila- - - - - - - - - - - - - - & - & - & - & - & - & - \\
\hline converal reduced game property & $+^{*}$ & $+^{*}$ & $+^{*}$ & - & + & - & - \\
- reduced game property & $+^{*}$ & + & + & + & - & - & -
\end{tabular}

Hence, the properties in Theorems 3, 4, and 6 are independent.

\section{References}

Arin, J. and E. Iñarra (2001). Egalitarian solutions in the core. International Journal of Game Theory, 30(2), 187-193.

Aumann, R. and M. Maschler (1985). Game theoretic analysis of a bankruptcy problem from the Talmud. Journal of Economic Theory, 36(2), 195-213.

Bondareva, O. and T. Driessen (1994). Extensive coverings and exact core bounds. Games and Economic Behavior, 6(2), 212-219.

Davis, M. and M. Maschler (1965). The kernel of a cooperative game. Naval Research Logistics Quarterly, 12, 223-259.

Dietzenbacher, B. (2018). Bankruptcy games with nontransferable utility. Mathematical Social Sciences, 92, 16-21.

Driessen, T. (1986). $k$-convex $n$-person games and their cores. Zeitschrift für Operations Research, 30(1), A49-A64.

Dutta, B. (1990). The egalitarian solution and reduced game properties in convex games. International Journal of Game Theory, 19, 153-169.

Gong, D., B. Dietzenbacher, and H. Peters (2021). Two-bound core games and the nucleolus. WorkingPaper 020, Maastricht University, Graduate School of Business and Economics, Netherlands. 
Maschler, M., B. Peleg, and L. Shapley (1971). The kernel and bargaining set for convex games. International Journal of Game Theory, 1(1), 73-93.

Muto, S., M. Nakayama, J. Potters, and S. Tijs (1988). On big boss games. The Economic Studies Quarterly, 39(4), 303-321.

O'Neill, B. (1982). A problem of rights arbitration from the Talmud. Mathematical Social Sciences, 2(4), 345-371.

Peleg, B. (1986). On the reduced game property and its converse. International Journal of Game Theory, 15(3), 187-200.

Potters, J., R. Poos, S. Tijs, and S. Muto (1989). Clan games. Games and Economic Behaviour, 1(3), 275-293.

Quant, M., P. Borm, H. Reijnierse, and B. van Velzen (2005). The core cover in relation to the nucleolus and the Weber set. International Journal of Game Theory, 33(4), 491-503.

Schmeidler, D. (1969). The nucleolus of a characteristic function game. SIAM Journal on Applied Mathematics, 17(6), 1163-1170.

Shapley, L. (1971). Cores of convex games. International Journal of Game Theory, 1, $11-26$. 